\title{
Cyclic Stretch Induces Vascular Smooth Muscle Cells to Secrete Connective Tissue Growth Factor and Promote Endothelial Progenitor Cell Differentiation and Angiogenesis
}

\author{
Jing Yan ${ }^{\dagger}$, Wen-Bin Wang ${ }^{\dagger}$, Yang-Jing Fan ${ }^{\dagger}$, Han Bao, Na Li, Qing-Ping Yao, \\ Yun-Long Huo, Zong-Lai Jiang, Ying-Xin Qi and Yue Han* \\ School of Life Sciences and Biotechnology, Institute of Mechanobiology and Medical Engineering, Shanghai Jiao Tong \\ University, Shanghai, China
}

OPEN ACCESS

Edited by:

Jing Zhou,

Peking University, China

Reviewed by:

Xiaohong Wang,

Tianjin Medical University, China

Suzanne Scarlata,

Worcester Polytechnic Institute,

United States

*Correspondence:

Yue Han

hanyue625@sjtu.edu.cn

†These authors have contributed equally to this work

Specialty section:

This article was submitted to

Cell Adhesion and Migration,

a section of the journal

Frontiers in Cell and Developmental

Biology

Received: 16 September 2020 Accepted: 10 November 2020 Published: 09 December 2020

Citation:

Yan J, Wang W-B, Fan Y-J, Bao H,

Li N, Yao Q-P, Huo Y-L, Jiang Z-L,

Qi Y-X and Han Y (2020) Cyclic

Stretch Induces Vascular Smooth Muscle Cells to Secrete Connective Tissue Growth Factor and Promote

Endothelial Progenitor Cell

Differentiation and Angiogenesis.

Front. Cell Dev. Biol. 8:606989.

doi: 10.3389/fcell.2020.606989
Endothelial progenitor cells (EPCs) play a vital role in endothelial repair following vascular injury by maintaining the integrity of endothelium. As EPCs home to endothelial injury sites, they may communicate with exposed vascular smooth muscle cells (VSMCs), which are subjected to cyclic stretch generated by blood flow. In this study, the synergistic effect of cyclic stretch and communication with neighboring VSMCs on EPC function during vascular repair was investigated. In vivo study revealed that EPCs adhered to the injury site and were contacted to VSMCs in the Sprague-Dawley (SD) rat carotid artery injury model. In vitro, EPCs were cocultured with VSMCs, which were exposed to cyclic stretch at a magnitude of $5 \%$ (which mimics physiological stretch) and a constant frequency of $1.25 \mathrm{~Hz}$ for $12 \mathrm{~h}$. The results indicated that stretched VSMCs modulated EPC differentiation into mature endothelial cells (ECs) and promoted angiogenesis. Meanwhile, cyclic stretch upregulated the mRNA expression and secretion level of connective tissue growth factor (CTGF) in VSMCs. Recombinant CTGF (r-CTGF) treatment promoted endothelial differentiation of EPCs and angiogenesis, and increased their protein levels of FZD8 and $\beta$-catenin. CTGF knockdown in VSMCs inhibited cyclic stretch-induced EPC differentiation into ECs and attenuated EPC tube formation via modulation of the FZD8/B-catenin signaling pathway. FZD8 knockdown repressed endothelial differentiation of EPCs and their angiogenic activity. Wnt signaling inhibitor decreased the endothelial differentiation and angiogenetic ability of EPCs cocultured with stretched VSMCs. Consistently, an in vivo Matrigel plug assay demonstrated that r-CTGF-treated EPCs exhibited enhanced angiogenesis; similarly, stretched VSMCs also induced cocultured EPC differentiation toward ECs. In a rat vascular injury model, r-CTGF improved EPC reendothelialization capacity. The present results indicate that cyclic stretch induces VSMC-derived CTGF secretion, which, in turn, activates FZD8 and $\beta$-catenin to promote both differentiation of cocultured EPCs into the EC lineage and angiogenesis, suggesting that CTGF acts as a key intercellular mediator and a potential therapeutic target for vascular repair.

Keywords: cyclic stretch, endothelial progenitor cells, differentiation, angiogenesis, vascular smooth muscle cells, connective tissue growth factor 


\section{INTRODUCTION}

Vascular injury leads to the initiation and progression of atherosclerotic vascular disease and may result in neointimal hyperplasia, in-stent restenosis, and acute stent thrombosis (Deanfield et al., 2007). Therefore, regeneration of the vascular endothelium is very important. Due to a low proliferative ability, the capacity of mature endothelial cells (ECs) to replace damaged endothelium is limited during vascular repair (Hristov et al., 2003; Deanfield et al., 2007). Accumulating studies indicate that bone marrow-derived endothelial progenitor cells (EPCs) play a crucial role in maintaining endothelial integrity. When damage occurs, EPCs are mobilized and home to the injury sites and then differentiate into ECs, which participate in angiogenesis, neovascularization, and tissue repair (Asahara et al., 1997; Deanfield et al., 2007; Zhang et al., 2014; Li et al., 2019). In this process, EPCs communicate with the exposed vascular smooth muscle cells (VSMCs), which compose the medial layer of the vessel wall. However, the impact of VSMCs on EPC function or the underlying signal mechanisms is little known.

Many studies have shown that EC and VSMC interaction in hemodynamic environments participates in the regulation of vascular stabilization, remodeling, and function, which suggests a role for mechanical forces generated by the pulsatile nature of blood pressure and flow in these processes (Chien, 2007; Qi et al., 2011; Chen et al., 2013; Deng et al., 2015). For example, VSMCs secrete exosomes enriched with miR-143$3 p$ that are transported to ECs, and endothelial proliferation is subsequently induced under pathologically elevated cyclic stretch (Deng et al., 2015). Mesenchymal stem cells promote the differentiation of EPCs into ECs via secreting VEGF and increasing expression of CD31 and vWF (Ge et al., 2018). These studies indicated that the cellular microenvironment and the resident cells might participate in determining EPC differentiation fate. Under physiological conditions, VSMCs are constantly subjected to mechanical stretching in vivo; therefore, we aimed to investigate whether mechanical forces and neighboring VSMCs modulate the endothelial differentiation of EPCs during endothelium repair.

Several studies have revealed that EPCs differentiate into either myocardial cells or endothelial cells, related to the cellular microenvironment of cytokines and coexisting cells (Murasawa et al., 2005; Ge et al., 2018). Resident cells contribute to the vascular repair process mostly via paracrine processes, secreting a mixture of growth factors and cytokines to recruit numerous cells to the injured sites and modulate the cell functions (Armulik et al., 2005; Ostriker et al., 2014). Connective tissue growth factor (CTGF), also known as CCN2, is a secreted protein of the CCN family, which includes Cyr61, CTGF, NOV, WISP1, WISP2, and WISP3 (Kubota and Takigawa, 2007; Yu et al., 2010; Jun and Lau, 2011; Liu et al., 2014). It has been reported that CTGF

Abbreviations: CTGF, connective tissue growth factor; DMEM, Dulbecco's modified Eagle medium; EPCs, endothelial progenitor cells; ECs, endothelial cells; EGM-2, endothelial growth medium-2; ELISA, enzyme-linked immunosorbent assay; FBS, fetal bovine serum; IPA, ingenuity pathway analysis; PET, polyethylene terephthalate; SD rats, Sprague-Dawley rats; siRNA, small interfering RNA; VSMCs, vascular smooth muscle cells. is implicated in vascular diseases, including atherosclerosis, hypertension, restenosis, and thrombosis. CTGF promotes neointimal hyperplasia after vascular injury and in rupture-prone atherosclerotic plaques; especially in areas of neovascularization and in the neointima in restenosis after balloon injuries, high CTGF expression levels are detected (Cicha et al., 2005; Kundi et al., 2009). It was also found that CTGF was strongly expressed in vascular cells, such as VSMCs, ECs, and fibroblasts (Jun and Lau, 2011). In our previous study, we found that CTGF is upregulated both in vivo and in vitro during hypertension, and $15 \%$ cyclic stretch significantly increased CTGF expression in VSMCs via microRNA-19b-3p, which indicated that CTGF is a key mechanical-sensitive molecule (Wang et al., 2019). Moreover, CTGF also plays a significant role in cell growth and differentiation. CTGF and VEGF induced pluripotent stem cells to differentiate into mature ECs (Kelly et al., 2020), and adiposederived stem cells can differentiate and proliferate with CTGF stimulation to enhance tendon repair (Li et al., 2019). However, the relationship between CTGF and EPCs has not been widely concerned and studied. Therefore, we hypothesized that VSMCderived CTGF contributes to the endothelial differentiation of EPCs in the process of vascular repair.

In this study, a rat vascular injury model and in vivo Matrigel plug assay were established to explore the effect of CTGF on EPC differentiation during vascular repair. An in vitro EPC//VSMC coculture system that included cyclic stretch was developed to detect the intercellular relationship between them. Here, we aimed to investigate whether CTGF is a key cell-to-cell interaction regulator for EPC differentiation in vascular repair.

\section{MATERIALS AND METHODS}

\section{Rat Carotid Artery Intimal Injury Model and Reendothelialization Assay}

Sprague-Dawley (SD) rats were anesthetized with isoflurane, and then the carotid arteries were exposed under the anatomical microscope. After the bifurcation above the common carotid artery was found, the occipital artery, the internal carotid artery, the thyroid artery, and the external carotid artery were ligated with a surgical suture in turn, and the thoracic segment of the common carotid artery was clamped with a hemostatic forceps. A balloon inserted $(0.67 \mathrm{~mm}$; Edwards Lifesciences, CA, USA) was applied to damage the intima through a small opening from the proximal end of the thyroid artery. EPCs $\left(1 \times 10^{6}\right)$ were pretreated with CM-Dil ( $1 \mu \mathrm{M}$, YEASEN, Shanghai, China) for $5 \mathrm{~min}$ at $37^{\circ} \mathrm{C}$ for labeling cells in red. Then, the cells were resuspended in $200 \mu \mathrm{l}$ of PBS and the suspension was instilled into and incubated with the freshly injured arterial bed for 2530 min (Griese et al., 2003; Yan et al., 2020). Postoperative rats continue to be housed with the experimental animal center. Recombinant CTGF ( $2 \mu \mathrm{g} / \mathrm{kg} /$ day) was firstly injected from the tail vein $3 \mathrm{~h}$ after injury and continued for 7 days. To observe the adhesion of EPCs, the left common carotid arteries were collected from the carotid bifurcation, incised longitudinally, and flattened between coverslips. The samples were fixed with $4 \%$ paraformaldehyde for $24 \mathrm{~h}$ and permeabilized with $0.3 \%$ Triton 
X-100 for 30 min. Nucleus was marked with DAPI $(1: 1,000)$ for $15 \mathrm{~min}$ at room temperature, and confocal microscopy (LV1000; Olympus, Tokyo, Japan) was used to take and analyze fluorescent images. Evans blue $(2 \%, 40 \mathrm{mg} / \mathrm{kg})$ was injected into the tail vein $30 \mathrm{~min}$ before sacrifice for the detection of reendothelialization assay on the seventh day after intimal injury. Photographs were taken with a digital camera. We quantified the reendothelialized parts that defined as areas not stained in blue with ImageJ software (Yan et al., 2020).

\section{Cell Culture and Identification}

VSMCs were harvested from the thoracic aorta of male SD rats by an explant technique (Qi et al., 2008, 2010). VSMCs were cultured in Dulbecco's modified Eagle medium (DMEM, Gibco) containing 10\% fetal bovine serum (FBS, Gibco) and incubated at $37^{\circ} \mathrm{C}$ with $5 \% \mathrm{CO}_{2}$. These cells were characterized by immunofluorescent staining, which was performed with an antibody recognizing smooth muscle specific $\alpha$-actin (Sigma). VSMCs from passages 4 to 8 were used in the following study.

EPCs derived from the bone marrow of SD rats were isolated and cultured as reported (Kuliszewski et al., 2009; Li et al., 2012). Briefly, total mononuclear cells were isolated from the tibias and femurs of male SD rats by density-gradient centrifugation with Histopaque-1083 (Sigma); the cells were then cultured in six-well plates with endothelial growth medium-2 (EGM-2) (Lonza) supplemented with fetal bovine serum (FBS), vascular endothelial growth factor (VEGF), fibroblast growth factor-B, epidermal growth factor, hydrocortisone, R3 insulin-like growth factor-1 (R3 IGF-1), ascorbic acid, and GA-1000 at $37^{\circ} \mathrm{C}$ in a $5 \% \mathrm{CO}_{2}$ incubator. The medium was changed after 4 days, and subsequent media changes were made every 3 days.

EPCs were identified by classical method (Asahara et al., 1997; Wei et al., 2015). The cells were incubated with FITCUEA-1 (Sigma) and Dil-Ac-LDL (Molecular Probes) as described previously. Double-positive staining for Dil-Ac-LDL and FITCUEA-I was considered an indication of EPCs. Surface markers for flow cytometry analysis include the stem cell markers CD133 and CD34, as well as the endothelial marker CD31. EPCs were stained with a panel of monoclonal antibodies including anti-CD133 (1:100, Proteintech), anti-CD34-PE (1:100, eBioscience), antiCD31-PE-Cy7 (1:100, eBioscience), and anti-CD45-PE (1:100, eBioscience). EPCs stained with anti-CD133 were incubated with an FITC-conjugated anti-mouse antibody. Unstained cells were incubated in parallel with antibody immunoglobulin (IgG) controls. Cells that were positive for CD133, CD34, and CD31 and negative for CD45 were considered to be EPCs. All samples were analyzed by flow cytometry (Becton Dickinson). EPCs from passages 2 and 3 were used (Li et al., 2018).

\section{EPC//VSMC Coculture Model and Cyclic Stretch Application}

EPCs $\left(1 \times 10^{5}\right.$ cells per well $)$ were seeded on the inner side of a cell culture insert (Becton Dickinson), which has a $10-\mu \mathrm{m}$-thick polyethylene terephthalate (PET) membrane containing $0.4-\mu \mathrm{m}$ pores. Flexible silicone bottom plates (Flexcell International) were either kept unseeded (EPC//O static group) or seeded with VSMCs $\left(2 \times 10^{5}\right.$ cells per well, EPC//VSMC static group). The insert with EPCs was incorporated into the flexible silicone bottom plates, which were loaded with an FX-5000T strain unit system (Flexcell International) for the application of cyclic stretch at 5\% stretch magnitude and $1.25 \mathrm{~Hz}$ frequency (Qi et al., 2016) for 6 or $12 \mathrm{~h}$ (EPC//VSMC stretch group) to mimic arterial mechanical conditions, as illustrated in Figure 2A.

\section{Western Blotting}

Western blotting was performed as described previously (Qi et al., 2008). Proteins were separated by 10\% SDSPAGE and transferred to PVDF membranes (Millipore, $0.22 \mu \mathrm{m})$. The membranes were incubated overnight with primary antibodies, respectively, against CTGF (1:800, Proteintech), FZD8 (1:1,000, Abcam), $\beta$-catenin (1:1,000, Cell Signaling Technology), Lamin A (1:1,000, Abcam), and GAPDH $\left(1: 1,000\right.$, Proteintech) at $4^{\circ} \mathrm{C}$, which was followed by immunoblotting with anti-rabbit or anti-mouse IgG $(1: 2,000$, Cell Signaling Technology) conjugated to HRP. Protein bands were detected by a standard enhanced chemiluminescence (ECL; Tanon) method and quantified by Quantity One software (Bio-Rad). Nuclear extracts were prepared by the Nuclear Extraction kit (Beyotime) according to the manufacturer's instructions.

\section{Quantitative Real-Time Polymerase Chain Reaction (QPCR)}

Total RNA was extracted from EPCs using Trizol reagent (Invitrogen) and was followed by cDNA synthesis using a RevertAid First Strand cDNA kit (Thermo Fisher Scientific). The resulting cDNA was used as a template in quantitative realtime polymerase chain reactions with SYBR Green Supermix on an ABI Prism 7500 sequence detection PCR system (Applied Biosystems) according to the manufacturer's protocol. The specific primer sequences are listed in Table 1. PCR conditions were as follows: $95^{\circ} \mathrm{C}$ for $30 \mathrm{~s}$ followed by 40 cycles at $95^{\circ} \mathrm{C}$ for $5 \mathrm{~s}, 60^{\circ} \mathrm{C}$ for $45 \mathrm{~s}$, and $72^{\circ} \mathrm{C}$ for $30 \mathrm{~s}$. The results were normalized to the GAPDH expression level, and relative quantification of the ratios was performed using the $2^{-\Delta \Delta \mathrm{Ct}}$ method.

TABLE 1 | The sequences of primers.

\begin{tabular}{lll}
\hline Gene & \multicolumn{2}{c}{ Primer sequences $\left(\mathbf{5}^{\prime} \mathbf{-} \mathbf{3}^{\prime} \mathbf{)}\right.$} \\
\hline CD31 & Fwd: GACAGCCAAGGCAGAT & Rev: ATTGGATGGCTTGGCC \\
& GCAC & TGAA \\
vWF & Fwd: GCGTGGCAGTGG & Rev: GGAGATAGCGG \\
& TAGAGTA & GTGAAATA \\
KDR & Fwd: GGCACCACTCAAA & Rev: CCTCTCTCCTCTC \\
& CGCTGAC & CCGACT \\
CTGF & Fwd: GGAAATGCTGTGAGG & Rev: TGTCTTCCAGTCGGT \\
& AGTGGGTT & AGGCAGCTA \\
GAPDH & Fwd: GGCACAGTCAAGG & Rev: ATGGTGGTGAAGA \\
& CTGAGAAT & CGCCAGTA
\end{tabular}

Fwd, forward; Rev, reverse. 


\section{Transfection of Small Interfering RNA}

The mRNA sequences of rat CTGF (NM_022266) were acquired from NCBI GenBank. Small interfering RNA (siRNA) against rat CTGF was designed and synthesized by GenePharma Biological Company (Shanghai, China). The sequences of the CTGF siRNAs were 5'-GGU CAA GCU GCC CGG GAA ATT-3' and 5'-UUU CCC GGG CAG CUU GAC CTT- $3^{\prime}$. The sequences of the FZD8 siRNAs were 5'-GGA AGU GAC CUC GCU ACU ATT-3' and 5'-UAG UAG CGA GGU CAC UUC CTT - $3^{\prime}$.

For the RNA interfering experiment, VSMCs were transfected with $100 \mathrm{nM}$ specific siRNA or scrambled siRNA with $5 \mu \mathrm{l}$ of Lipofectamine $^{\mathrm{TM}} 2000$ (Invitrogen) in Opti-MEM (Gibco) for $48 \mathrm{~h}$ following the manufacturer's instructions.

\section{Matrigel Tube Formation Assay}

An in vitro tube formation assay was performed as previously described (Malinda, 2009). In brief, Matrigel (Corning) was added to 24-well plates at $300 \mu \mathrm{l}$ per well and to polymerize at $37^{\circ} \mathrm{C}$ for $30 \mathrm{~min}$. Then, $2 \times 10^{4} \mathrm{EPCs}$ were seeded onto the coated wells and cultured with $500 \mu \mathrm{lEBM}-2$ at $37^{\circ} \mathrm{C}$ with $5 \% \mathrm{CO}_{2}$ for $6 \mathrm{~h}$. EPC tube formation was assessed by microscopy, and photographs were randomly taken of five fields. The total tube length was calculated using imaging analysis software (Image-Pro Plus 6.0).

\section{In vivo Matrigel Plug Angiogenesis Assay}

An in vivo angiogenesis assay was performed as previously described (Han et al., 2016). EPCs were treated with or without recombinant CTGF (r-CTGF; $20 \mathrm{ng} / \mathrm{ml}$ ) for $24 \mathrm{~h}$. Growth factorreduced Matrigel (400 $\mu \mathrm{l} / \mathrm{plug})$ was thawed and mixed with EPCs $\left(1 \times 10^{6} / \mathrm{plug}\right)$, and Matrigel supplemented only with $\mathrm{r}$ CTGF in the absence of EPCs was used as a blank control. In addition, to investigate how VSMCs and stretch affect EPC differentiation, EPCs were cultured by themselves under static conditions or cocultured with VSMCs, which had cyclic stretch applied at 5\% stretch magnitude and $1.25 \mathrm{~Hz}$ frequency. After $12 \mathrm{~h}$, EPCs under different conditions were harvested by treatment with $0.25 \%$ trypsin and were mixed with Matrigel. Each plug was subcutaneously injected into the flank of 6-week-old male nude mice ( $n=4$ in each group). Mice were euthanized 7 days after surgery. Matrigel plugs were harvested, fixed in $10 \%$ formalin, and embedded in paraffin; multiple $5-\mu \mathrm{m}$-thick slices were prepared. Immunofluorescent staining was performed using an anti-CD31 antibody (Abcam) and anti-CD34 antibody (Santa Cruz).

\section{Enzyme-linked Immunosorbent Assay (ELISA)}

The concentration of CTGF in cell culture medium from VSMCs was determined by enzyme-linked immunosorbent assay by using a rat CTGF ELISA kit (Cloud-Clone Corp). The assay was performed according to the manufacturer's instructions.

\section{Ingenuity Pathway Analysis}

Ingenuity Pathway Analysis (IPA) software (Qiagen) was used to search for the genes that possibly interact with CTGF. The software predicted the downstream genes of specific target through Grow Tool. After prediction, the possible canonical pathways and functional classifications of the target genes of CTGF were obtained with IPA. The significance values for analyses of network and pathway generation were calculated using the right-tailed Fisher's Exact Test by comparing the number of proteins that participate in a given function or pathway relative to the total number of occurrences of these proteins in all functional/pathway annotations stored in the Ingenuity Pathway Knowledge Base (IPKB). IPA was used to understand the complex biological and chemical systems at the core of life science research based on lectures or predicated analysis (Dai et al., 2009).

\section{Statistical Analysis}

Data are expressed as the mean $\pm S D$ from at least three independent experiments. Statistical analysis was performed with Student's $t$-tests for two groups of data and with one-way ANOVA for multiple comparisons, followed by Bonferroni's post-hoc test. Non-parametric data were analyzed with the Mann-Whitney $t$ test in GraphPad Prism software. Values of $P$ that were $<0.05$ were considered statistically significant.

\section{RESULTS}

\section{The Adhesion of EPCs at Intimal Injury Site in vivo}

We established a carotid artery intimal injury model that is shown in Figure 1A to explore the potential interaction between EPCs and VSMCs. The left and right common carotid artery of each rat were used as the experimental group and autologous control, respectively. After 4 weeks, hematoxylin-eosin (HE) staining results indicated that vessel wall was significantly thickened, which revealed the intimal hyperplasia (Figure 1B). To confirm that the intimal injury model was successfully established, we harvested the injury and control vessels right after the surgery. Immunofluorescence staining results showed that the von Willebrand factor (vWF), a marker of the ECs marked in red, was not detected on the inner wall of vessels right after the injury surgery, indicating that the model was successfully established (Figure 1C). In the initial stage of intimal injury, EPCs are mobilized to the injury site; hence, we then examined the adhesion of EPCs $1 \mathrm{~h}$ after intimal injury, which were identified by vWF (EC marker) and CD34 (stem cell marker) expression. Due to the balloon injury, the endothelial layer was damaged and did not express vWF, hence, the co-expression of CD34 (red) and vWF (green) indicated the adhesion of EPCs at the injury site (Figure 1D). In vivo experiment results suggested that carotid intimal injury caused EPCs homing and adhering to the injury site, which were connected to the exposed VSMCs, which are subjected to physiological stretch at the damaged intima. Hence, we hypothesized that the intercellular communication between EPCs and VSMCs might have an effect on EPC function.

\section{Stretched VSMCs Promoted the Endothelial Differentiation of Cocultured EPCs}

To examine the effect of VSMCs and physiological cyclic stretch on EPC function, an EPC//VSMC coculture with a cyclic stretch 
A
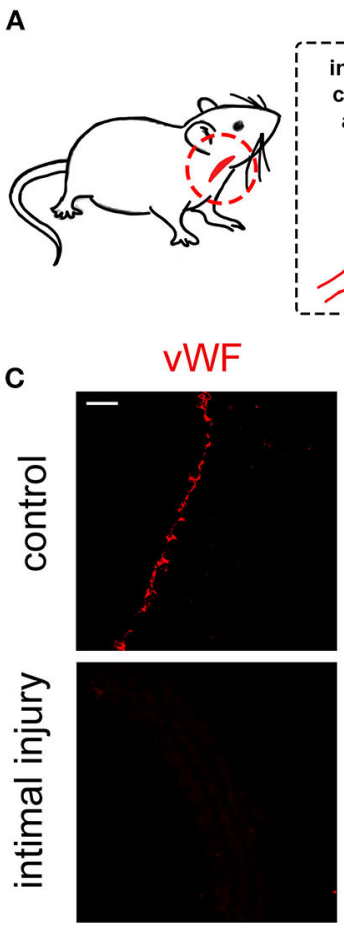

D
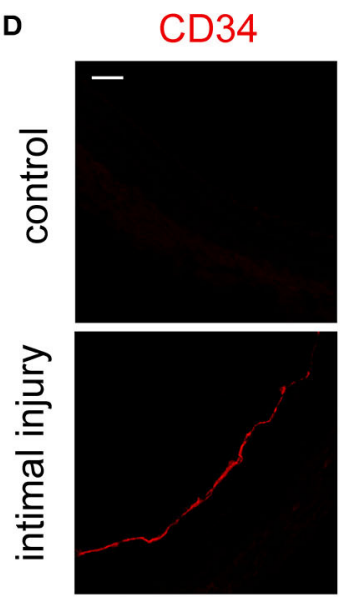

\section{B}

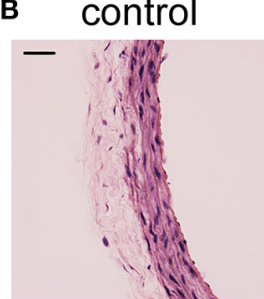

DAPI
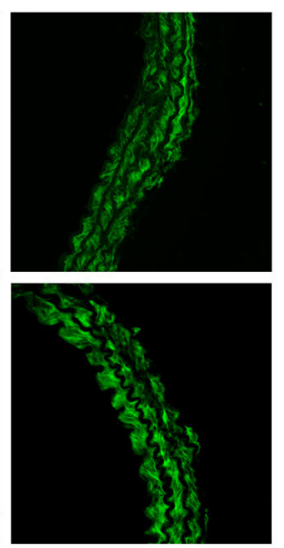

vWF
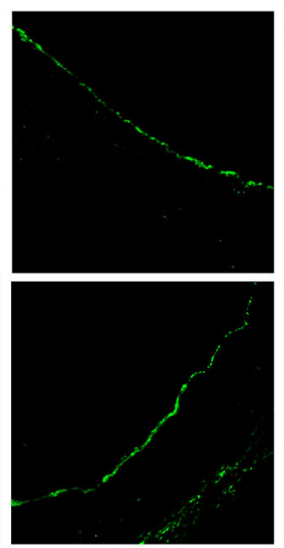
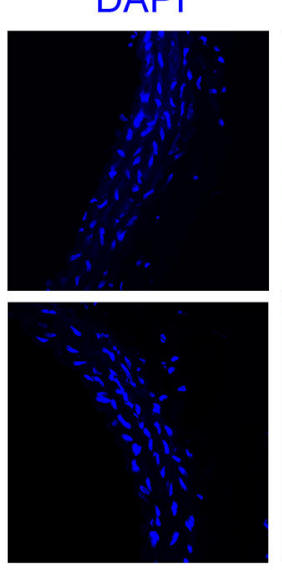

DAPI
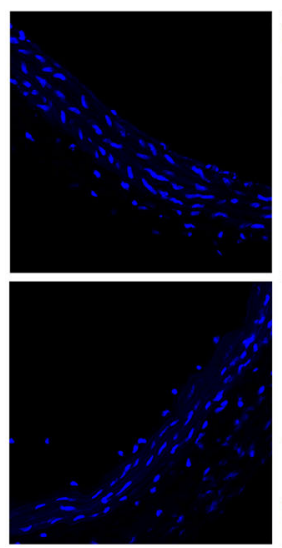

intimal injury
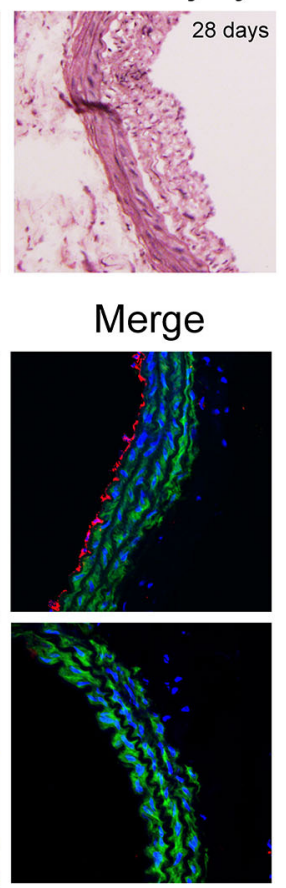

Merge
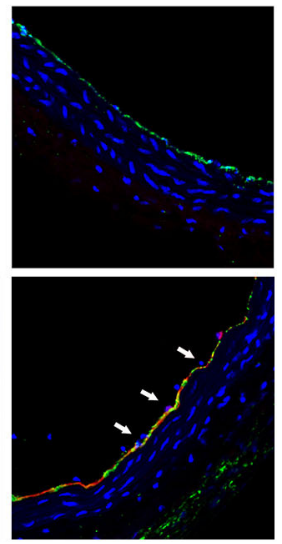

FIGURE 1 | EPCs adhered to the injury site $1 \mathrm{~h}$ after balloon damaged the intimal. (A) Schematic diagrams of the establishment of rat intimal balloon damage model. (B) HE staining indicated the significant intimal hyperplasia and the thickening blood vessel wall after 28 days compared with control group $(n=4)$. (C) We harvested the vessels for the immunofluorescence staining right after the surgery. The immunofluorescence staining results showed that vWF (red) was not expressed after the balloon injury, indicating that the intima fell off and the model was successfully constructed. The $\alpha$-SMA (green) and DAPI (blue) were used to identify VSMCs and nuclei, respectively $(n=4)$. Scale bar $=50 \mu \mathrm{m}$. (D) The adhesion of EPCs in situ $1 \mathrm{~h}$ after intimal injury was identified by CD34 (stem cell marker, red) and vWF (EC marker, green) by immunofluorescence staining. The results showed that CD34 and vWF were co-expressed at same place, indicating that EPCs adhered to the damaged endothelial layer. The results showed that CD34 and VWF were colocalized, indicating that EPCs adhered to the inner wall of blood vessels. The staining of VWF in the control group was due to the presence of ECs. Nuclei are stained in blue by DAPI. The white arrows indicate the adhered EPCs at the injury site $(n=4)$. Scale bar $=50 \mu \mathrm{m}$.

application system was used (Figure 2A). EPCs were isolated from SD rat bone marrow by density gradient centrifugation and identified by fluorescence microscopy and flow cytometry. EPCs were characterized as adherent cells that were double positive for Dil-acLDL uptake and FITC-UEA-lectin binding (Figure 2B). Moreover, these cells were found to be positive for CD31, CD34, and CD133 but negative for CD45 by flow cytometric analysis (Figure 2B). VSMCs were identified by immunofluorescent staining with $\alpha$-smooth muscle actin (SMA), which is shown in green (Supplementary Figure 1).

In the static system, the mRNA levels of the EC markers CD31, vWF, and KDR in EPCs cocultured with VSMCs showed no significant change at $6 \mathrm{~h}$ (Figure 2C) or $12 \mathrm{~h}$ (Figure 2D), compared to the levels observed in monocultured EPCs. 
A

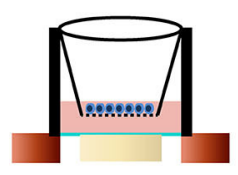

EPC//O

B

(a)

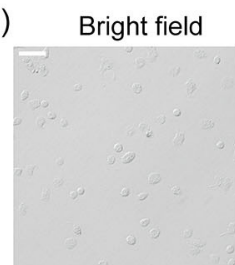

FITC-UEA
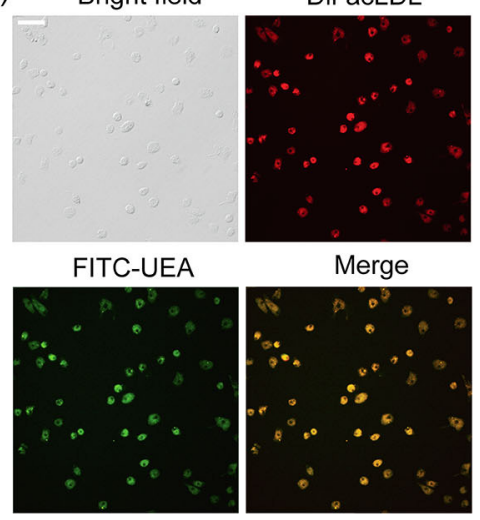

EPC/NSMC

Static

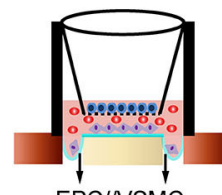

EPCINSMC

Stretch

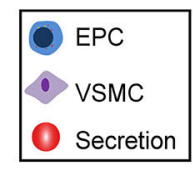

(b)
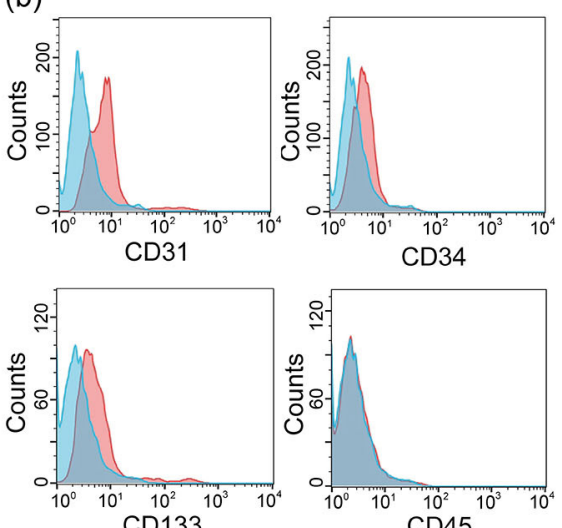

D

D $\quad$...... EPC//O static

C
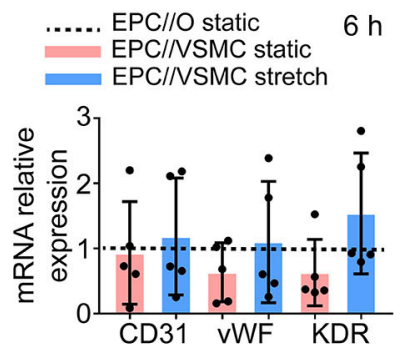

EPC/NSMC static

EPC/NSMC stretch

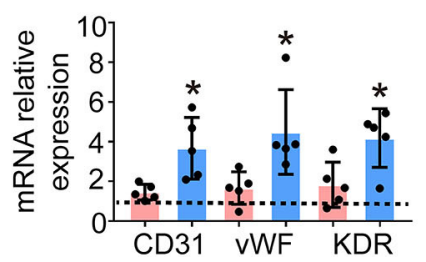

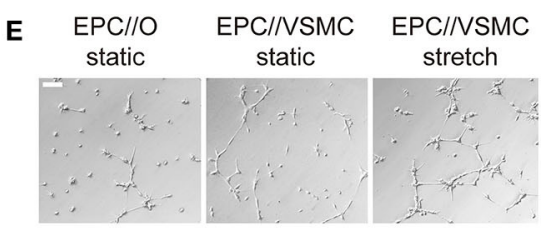

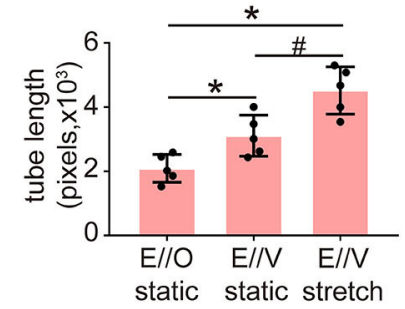

$\mathbf{F}$

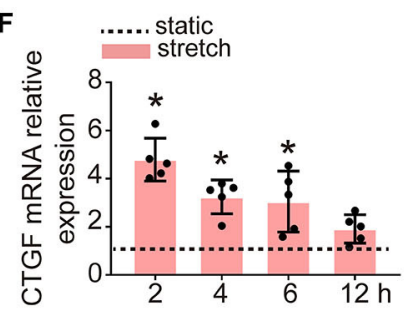

FIGURE 2 | Five percent cyclic stretch induces VSMC-derived CTGF secretion and promotes the differentiation of cocultured EPCs into ECs. (A) Schematic diagrams show EPC/NSMC coculture and the cyclic stretch system. (B) EPCs showed a spindle-shaped morphology after 8 days. Staining of FITC-UEA-lectin (green) and Dil-acLDL (red) revealed double-positive cells that were identified as EPCs (a). FACS analysis showed that EPCs were positive for the endothelial cell marker CD31 and hematopoietic stem cell markers CD34 and CD133, and they were negative for the leukocyte marker CD45. Controls (blue area) were overlaid on the histogram of each surface antigen (red areas) tested (b). (C) The mRNA levels of the EC markers CD31, vWF, and KDR showed no differences in EPCs among different cocultured conditions after $6 \mathrm{~h}(n=5)$. Monocultured EPCs under static were used as control groups, shown as the dotted line. (D) The mRNA levels of the EC markers CD31, VWF, and KDR were induced in EPCs cocultured with stretched VSMCs after $12 \mathrm{~h}(n=5)$. (E) EPCs cocultured with stretched VSMCs had an increased tube formation ability after $12 \mathrm{~h}(n=5)$. (F) QPCR results revealed that CTGF mRNA levels were increased at different time points in stretched VSMCs ( $n=5)$. (G) The level of CTGF secretion from stretched VSMCs was significantly elevated at $12 \mathrm{~h}(n=5)$. For quantitative analysis, five fields per plate were photographed, and tube lengths were measured using Image-Pro Plus software. Scale bar $=100 \mu \mathrm{m}$. Values are expressed as the mean $\pm S D$. * $\# P<0.05$ compared with the respective control.

Statistical analysis was performed with Student's $t$-test and one-way ANOVA for (C-G).

However, once the VSMCs were subjected to $5 \%$ cyclic stretch for $12 \mathrm{~h}$, the expression of EC markers in cocultured EPCs was significantly increased as compared with monocultured EPCs (Figure 2D). To determine the role of stretched VSMCs in the angiogenic capability of EPCs, Matrigel tube formation assays were performed. As shown in Figure 2E, the total length of tubelike structures was significantly greater in EPCs cocultured with VSMCs under 5\% cyclic stretch than in the static cocultured (E//V, static) or monocultured EPCs (E//O, static) at $12 \mathrm{~h}$. These results indicated that the differentiation of EPCs is regulated by paracrine factors secreted from stretched VSMCs.

In addition, to explore the effect of cyclic stretch on CTGF expression in VSMCs, we detected CTGF expression levels at different time points. The results revealed that both the mRNA and secretion levels of CTGF were elevated in VSMCs exposed to $5 \%$ cyclic stretch in vitro (Figures 2 F,G). The results suggested that CTGF may participate in stretched VSMCinduced EPC differentiation.

\section{CTGF Induced EPC Differentiation Toward ECs in vitro}

To investigate whether CTGF modulates EPC differentiation and angiogenesis, EPCs were treated with r-CTGF. We stimulated EPCs with different doses of r-CTGF protein $(0,5,10,15$, 20 , and $30 \mathrm{ng} / \mathrm{ml}$ ) for $24 \mathrm{~h}$ under static conditions (Figure 3A). Exogenous CTGF at a concentration of $20 \mathrm{ng} / \mathrm{ml}$ promoted the 

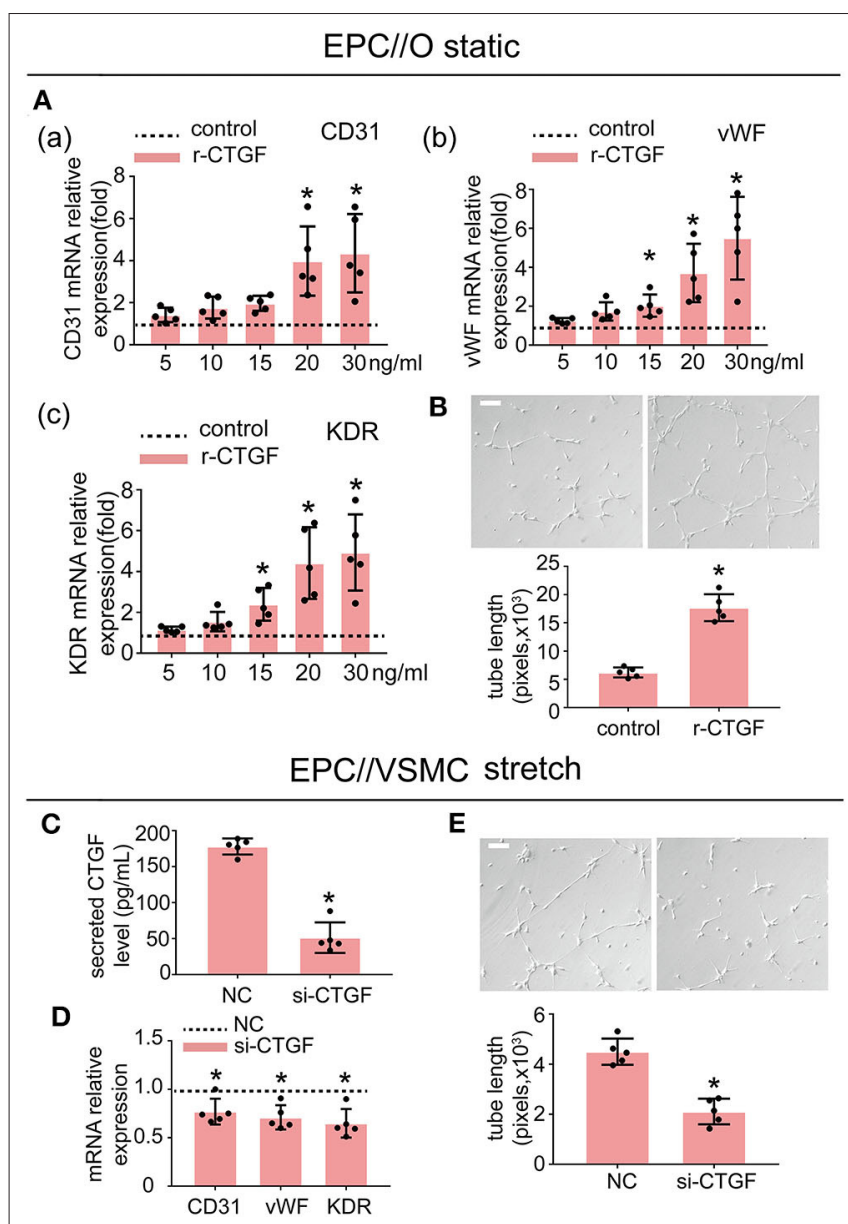

FIGURE 3 | r-CTGF induced EPC differentiation into ECs under static conditions; stretched VSMCs modulate the differentiation of EPCs via CTGF in vitro. (A) EPCs were treated with different doses of r-CTGF $(5,10,15,20$, and $30 \mathrm{ng} / \mathrm{ml}$ ) for $24 \mathrm{~h}$, and the mRNA expression of EC markers CD31, vWF, and $\mathrm{KDR}$ were significantly increased at a concentration of $20 \mathrm{ng} / \mathrm{ml}$ and $30 \mathrm{ng} / \mathrm{ml}$ $(n=5)$. EPCs treated without $r-C T G F$ were used as control groups. (B) EPCs treated with $\mathrm{r}$-CTGF $(20 \mathrm{ng} / \mathrm{ml})$ for $24 \mathrm{~h}$ significantly exhibited increased tube formation $(n=5)$. (C) CTGF siRNA was transfected under $5 \%$ cyclic stretch conditions, and the CTGF secretion level was significantly decreased in VSMCs. (D,E) Transfection with CTGF siRNA under 5\% cyclic stretch conditions with cocultured EPCs resulted in the inhibition of EPC differentiation toward ECs and decreased their tube formation $(n=5)$. Scale bar $=100 \mu \mathrm{m}$. For quantitative analysis, five fields per plate were photographed, and tube lengths were measured using Image-Pro Plus software. Values are expressed as the mean $\pm S D$. ${ }^{*} P<0.05$ compared with the respective control. Statistical analysis was performed with Student's t-test for (A-E).

expression of the EC markers CD31, vWF, and KDR remarkably in EPCs. Meanwhile, r-CTGF at the concentration of $20 \mathrm{ng} / \mathrm{ml}$ promoted EPC tube formation (Figure 3B). To further determine the role of CTGF, its expression was knocked down in VSMCs by CTGF-specific siRNA. After transfected with CTGF siRNA for $48 \mathrm{~h}, \mathrm{VSMCs}$ were then subjected to cyclic stretch. The effect of CTGF siRNA on the secretion level of CTGF was significantly decreased in VSMCs (Figure 3C). CTGF knockdown in stretched VSMCs could reverse EPC differentiation and angiogenesis capabilities (Figures 3D,E) in comparison with the control group. Overall, the results indicated that CTGF plays a key role in stretched VSMC-induced EPC differentiation in vitro.

\section{The Potential Downstream Targets of CTGF Were Predicted by Ingenuity Pathway Analysis}

CTGF is essential for the differentiation of EPCs; however, the underlying mechanism involved remains unclear and needs to be further studied. By using ingenuity pathway analysis (IPA) with high prediction criteria, the top 20 molecules that are potentially downstream of CTGF were selected (Figure 4A). Then, IPA analysis allowed further insights into the cellular functions (Figure 4B) and canonical pathways (Figure 4C) of the top 20 genes. The top molecular and cellular function was cellular development, and the top canonical pathway that was identified was the Wnt/ $\beta$-catenin signaling pathway, which plays an essential role in developmental decisions and regulating progenitor cell fate during embryonic development. According to reports, FZD8, a member of Frizzled receptors located on the cell membrane, functions as a key component of the Wnt signaling pathway (Chakravarthi et al., 2018; Murillo-Garzon et al., 2018). When Wnt proteins bind to transmembrane FZD8 receptors and coreceptors, $\beta$-catenin-dependent signals can be activated (Murillo-Garzon et al., 2018). Thus, we hypothesized that FZD8 and $\beta$-catenin, which are key regulators of the $\mathrm{Wnt} / \beta$ catenin signaling pathway, might participate in the process of CTGF-induced EPC differentiation.

\section{CTGF Regulated FZD8/ $\beta$-catenin Expression in EPCs}

To further determine the effect of CTGF on FZD8 and $\beta$-catenin expression, EPCs were treated with r-CTGF in vitro. Western blot results revealed that $\mathrm{r}$-CTGF induced the expression of FZD8 (Figure 5A). Moreover, the protein level of $\beta$-catenin was increased in both nuclear and cytosolic lysates from EPCs (Figure 5B). As shown in Figure 5C, r-CTGF treatment leads to significant increases of $\beta$-catenin in both the cytoplasm and the nucleus of EPCs. To further determine the role of CTGF under cyclic stretch conditions, its expression was knocked down in VSMCs by CTGF-specific siRNA. VSMCs were treated with CTGF siRNA for $48 \mathrm{~h}$ and then subjected to cyclic stretch. In contrast to the effect of r-CTGF, knockdown of CTGF in VSMCs not only suppressed FZD8 (Figure 5D) and $\beta$-catenin (Figure 5E) protein expression but also decreased the expression of $\beta$-catenin in both the cytoplasm and the nucleus of EPCs (Figure 5F). Taken together, these findings suggest that CTGF may modulate EPC differentiation and angiogenesis via the FZD8 and $\beta$-catenin pathways.

To better understand the role of FZD8 and Wnt signaling in regulating EPC differentiation, EPCs were treated with an FZD8-specific siRNA and with XAV-939 (specific inhibitor of Wnt signaling), respectively. The effect of the siRNA treatments on FZD8 mRNA levels was assessed (Supplementary Figure 2). The results indicated that FZD8 knockdown in EPCs could decrease angiogenic ability (Figure 6A) and could decrease EPC differentiation into the EC lineage (Figure 6B). To confirm the 

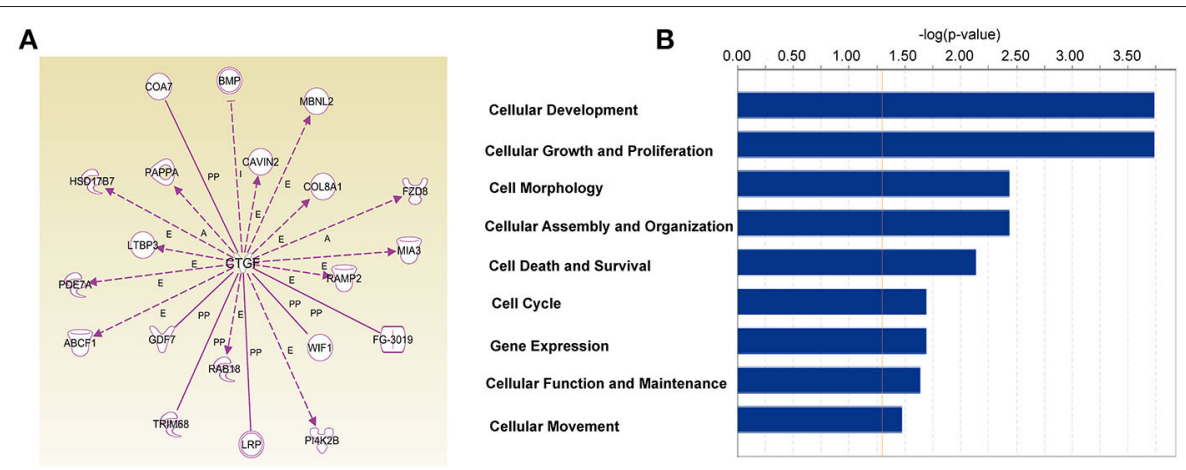

C

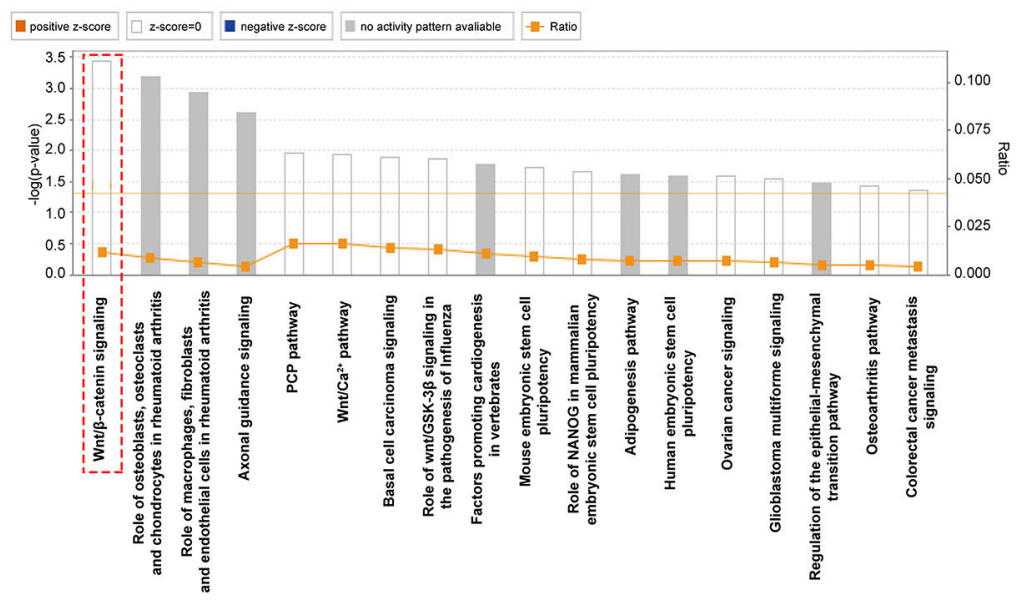

FIGURE 4 | The potential downstream targets of CTGF that were predicted by IPA are shown. (A) Molecules predicted to interact with CTGF were determined using IPA software. IPA analysis showed that downstream targets are involved in molecular and cellular functions (B) and canonical pathways (C).

role of Wnt signaling in cyclic stretch-induced actions of CTGF on EPCs, the inhibitor of Wnt signaling, XAV-939, was used to treat cocultured EPCs at the concentration of $5 \mu \mathrm{M}$. XAV-939 treatment remarkably inhibited EPC tube formation (Figure 6C). Meanwhile, XAV-939 treatment decreased the expression of the EC markers CD31, vWF, and KDR in EPCs (Figure 6D).

\section{CTGF Promoted the Differentiation of EPCs in vivo}

To examine the role of CTGF in EPC differentiation in vivo, the Matrigel plug assay was performed. In this assay, EPCs with or without r-CTGF treatment were mixed with Matrigel and subcutaneously injected into the flank of nude mice, and Matrigel supplemented only with r-CTGF in the absence of EPCs was used as a blank control. On day 7 , mice were sacrificed, and Matrigel plugs were harvested (Figure 7A). Abundant vessel formation was shown in the plugs of EPCs treated with $r$ CTGF compared with what was observed in the controls. In addition, few cells invaded the plug in the Matrigel that was injected r-CTGF in the absence of EPCs. The Matrigel plugs were embedded in paraffin to enable histological analysis and the level of EPC differentiation was detected using an antibody against the EC marker CD31. We observed a significant increase in CD31 staining in the group of r-CTGF-treated EPCs, and there was no CD31 staining in the sections of plugs mixed with $r$ CTGF and no EPCs (Figure 7B). To further demonstrate that r-CTGF promoted endothelial differentiation of EPCs in vivo, we also performed the stem cell marker CD34 immunofluorescence staining. CD34 staining results showed that protein expression levels in EPCs treated with r-CTGF were significantly reduced compared to the control group (Figure 7B). The results indicated that CTGF enhanced the differentiation of EPCs into ECs in vivo.

Furthermore, to investigate how VSMCs and stretch affect EPC differentiation, another Matrigel plug assay was also performed. EPCs were monocultured under static conditions or cocultured with VSMCs that were subjected to cyclic stretch at 5\% stretch magnitude and $1.25 \mathrm{~Hz}$ frequency. After $12 \mathrm{~h}$, EPCs under different conditions were harvested and mixed with growth factor-reduced Matrigel. Matrigel was injected subcutaneously into the flank of nude mice. On day 7, the Matrigel plugs were harvested after mice were sacrificed. The results show that there is a significant increase in CD31 staining and decrease in CD34 in the plugs mixed with cocultured EPCs under cyclic stretch conditions in comparison to the group with monocultured EPCs under static conditions; these results indicate that VSMCs and stretching prompt the differentiation of EPCs into the endothelial lineage (Figure 7C). 


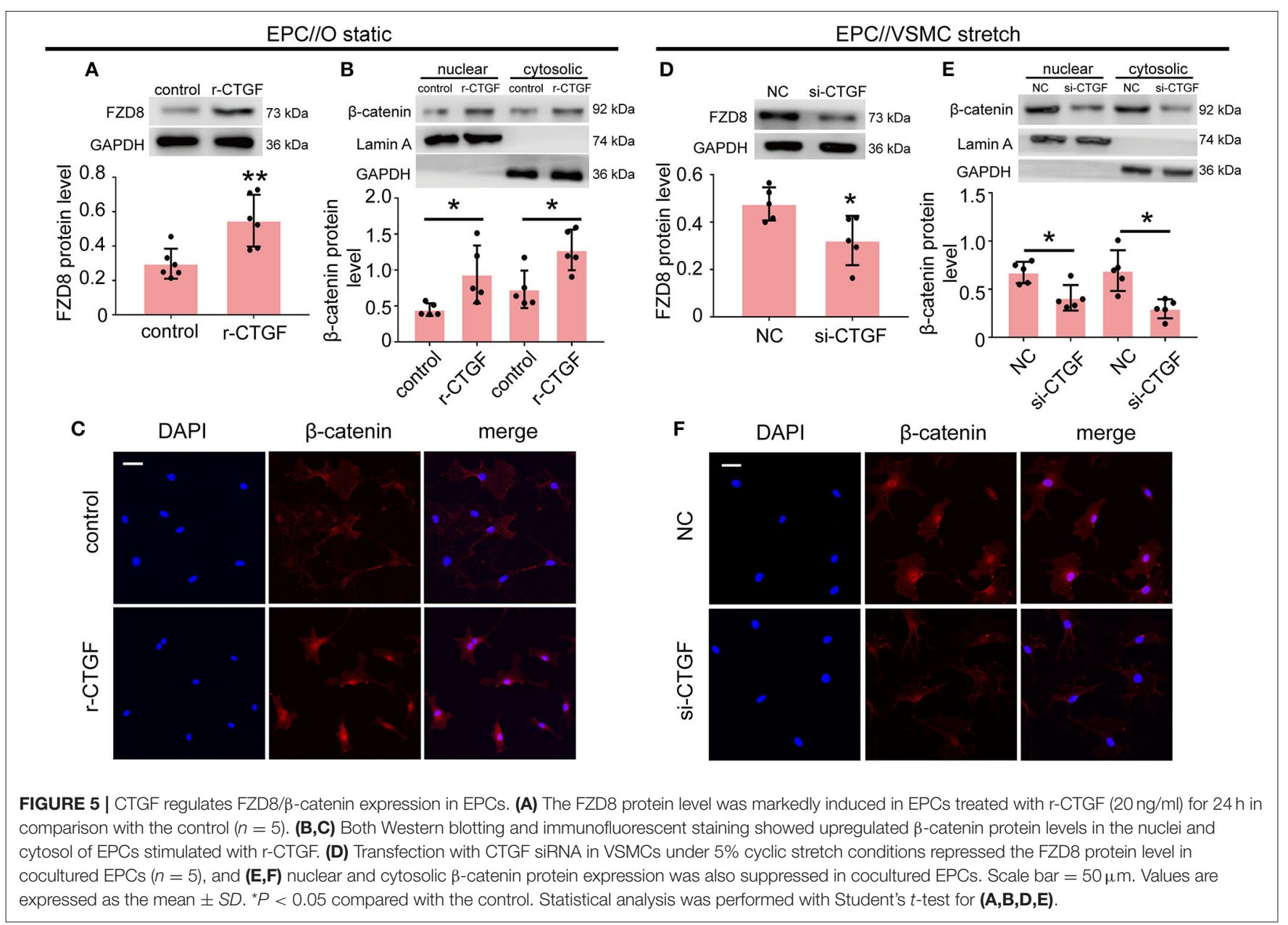

\section{CTGF Promoted Reendothelialization Capacity of EPCs After Intimal Injury in vivo} To further verify the effect of CTGF on EPC differentiation after intimal injury, the rat carotid intimal injury model was established. After carotid intimal injury, CM-Dil-labeled EPCs were incubated at the fresh injury site. The adherent EPCs directly contacted with VSMCs, and meanwhile, both cells were under cyclic stretch. The degree of reendothelialization after vascular injury is widely recognized and used in the detection of differentiation of EPCs and the repair of intimal injury by Evans blue dye. The flow diagram of the experimental protocol was shown in Figure 8A. Immunofluorescence staining results indicated that CM-Dil-labeled EPCs adhered to the vascular lesion in situ (Figure 8B). The rat left carotid artery without surgery was imaged in Figure 8C. Evans blue staining was used to detect reendothelialization after intimal injury. Compared with the sham operation group, the experimental group treated with PBS injection only had the largest blue area. The blue area decreased slightly after systemic injection of $r$ CTGF, indicating an increase of vascular reendothelialization. Furthermore, after intimal injury, local incubation with EPCs significantly increased intimal repair, and tail vein injection of r-CTGF for 7 days significantly promoted reendothelialization capacity of EPCs and upregulated reendothelialization area (Figure 8D).

\section{DISCUSSION}

In this study, we characterized the so far unknown synergistic effect of physiological cyclic stretch and communication with neighboring VSMCs on endothelial differentiation of EPCs and their angiogenic activity. Our results highlight and help to better understand the role of mechanical stretch and regulation of intracellular communication in vascular repair, which indicate that cyclic stretch induces VSMC-derived CTGF secretion, which in turn activates FZD8 and $\beta$-catenin to promote both the endothelial differentiation of cocultured EPCs and angiogenesis.

Increasing reports have shown that EPCs, which quiescently reside in physiological bone marrow and can be recruited to sites of injury, are important for maintaining endothelial integrity and take part in the process of re-endothelization, neovascularization, and wound healing by differentiating into mature ECs and secreting angiogenic factors when endothelial injury occurs (Hristov et al., 2003; Hur et al., 2004; Zhang et al., 2014). Previous studies have revealed that the differentiation fate of EPCs is affected by many factors, such as mechanical 


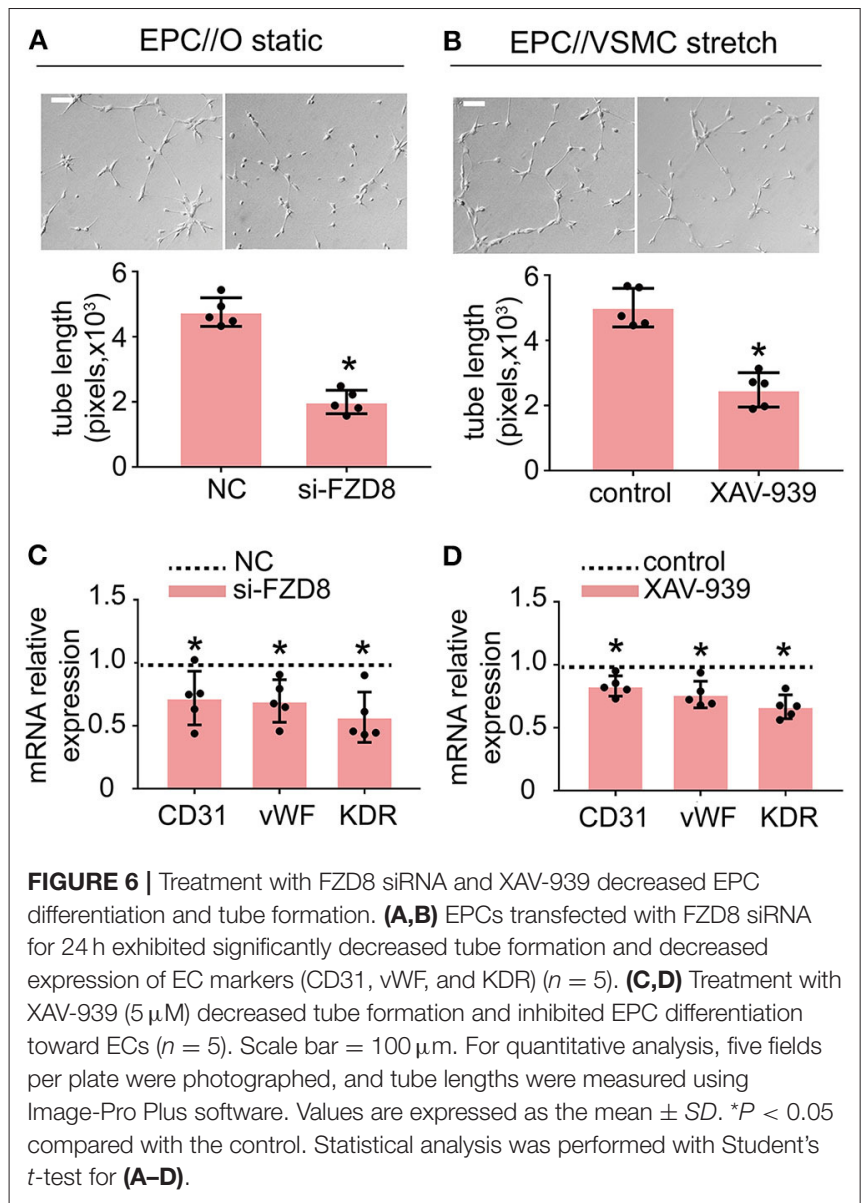

forces (including shear stress and cyclic stretch) and angiogenic factors (such as VEGF and CCN61) (Yu et al., 2010; Cheng et al., 2014; Li et al., 2017, 2018). Mesenchymal stem cells promote the endothelial differentiation of EPCs with the upregulation of CD31 and vWF (Ge et al., 2018). It has been reported that smooth muscle progenitor cells were reported to promote the neovascularization of EPCs and enhance the efficiency of EPC therapy during the wound-healing process (Joo et al., 2014; Foubert et al., 2015). Shudo et al. showed that the normal interactions between VSMCs and EPCs increased functional microvasculature and enhanced myocardial function in a rodent ischemic cardiomyopathy model (Shudo et al., 2013). However, the influences of neighboring VSMCs and physiological stretch on EPC functions are unknown. In this study, we developed a coculture system that assured intimate cellular communication between EPCs and VSMCs and included the application of physiological mechanical stretching. The present findings show that VSMCs facilitate EPC differentiation into ECs that have elevated expression of EC markers (CD31, vWF, and KDR) and increased angiogenic ability in the presence of mechanical stretch, indicating the importance of the synergistic effect of VSMCs and mechanical forces on EPC function.

In the present study, the Flexcell FX-5000T Strain Unit was applied to generate mechanical stretch in vitro. The $5 \%$ cyclic stretch (mimicking the physiological cyclic stretch) magnitude is based on previous clinical ultrasound data, which indicated that the large artery dilates by as much as $5 \%$ during cyclic stretch in normotension (Asanuma et al., 2003; Maul et al., 2011). Furthermore, the frequency used in the present study simulates the adult heart rate (70-80 beats) (Wachowiak et al., 2016), and some studies on rat VSMC cultures use an even lower frequency. The magnitude and frequency used here have also been used in our previous publications (Qi et al., 2016; Yao et al., 2017).

It has been shown that mechanical stretching increased the amount of CTGF secretion in anterior cruciate ligamentderived cells (Miyake et al., 2011), indicating that CTGF is a mechano-sensitive molecule. Blomme et al. reported that valvular interstitial cells showed the significant upregulation of CTGF at $1.15 \mathrm{~Hz}$ (heart frequency) and $14 \%$ elongation mechanical stretch, which was promoted through RhoC and MEK/Erk signaling pathways (Blomme et al., 2019). Additionally, in our previous study, we found that pathological cyclic stretch induces vascular remodeling by promoting VSMC proliferation via the miR-19b-3p/CTGF pathway (Wang et al., 2019).

Accumulating evidence indicates that CTGF, as a potent angiogenic inducer, supports cell metastasis, invasion, and angiogenesis in several tumor cells, and it induces tube formation in ECs (Kubota and Takigawa, 2007). It suggests that there is probably an interaction between angiogenesis of EPCs and CTGF. Our previous results indicated that 5\% cyclic stretch induced the expression of CTGF in VSMCs; hence, in the current study, we aimed to investigate whether stretch-induced CTGF plays a role in EPC function. The results showed that $\mathrm{r}$-CTGF significantly enhanced the expression of endothelial markers at the mRNA level and enhanced tube formation, whereas VSMCs transfected with a CTGF-specific siRNA suppressed this expression. Meanwhile, by using IPA bioinformatics analysis, FZD8 was found to be a putative downstream target regulated by CTGF.

FZD8 and $\beta$-catenin are key regulators in the $\mathrm{Wnt} / \beta$ catenin signaling pathway that have essential roles in the regulation of progenitor cell fate, adult tissue homeostasis, proliferation during embryonic development, and developmental decisions (Majidinia et al., 2018). For example, ATP activates $\mathrm{Wnt} / \beta$-catenin signaling in mesenchymal stem cells (MSCs), which contributes to neuronal differentiation by inducing the expressing of neuronal markers, Tuj1 and $\mathrm{NeuN}$ (Tu et al., 2014). Attenuation of $\mathrm{Wnt} / \beta$-catenin signaling by exposure to the epidermal growth factor receptor is required for hair follicle development (Tripurani et al., 2018). In addition, during retinal neovascularization in oxygen-induced retinopathy, the Wnt/ $\beta$-catenin signaling pathway had an important role in EPC recruitment (Liu et al., 2013). In our study, the positive effect of CTGF on FZD and $\beta$-catenin expression was detected. Therefore, CTGF may modulate the differentiation fate and angiogenic ability of EPCs by activating the FZD8/ $\beta$-catenin pathway. Moreover, to better understand the regulation mechanism, we further detected the FZD8 mRNA level of EPCs after the r-CTGF stimulation. Results showed that the mRNA level was significantly increased by r-CTGF, which suggested that 


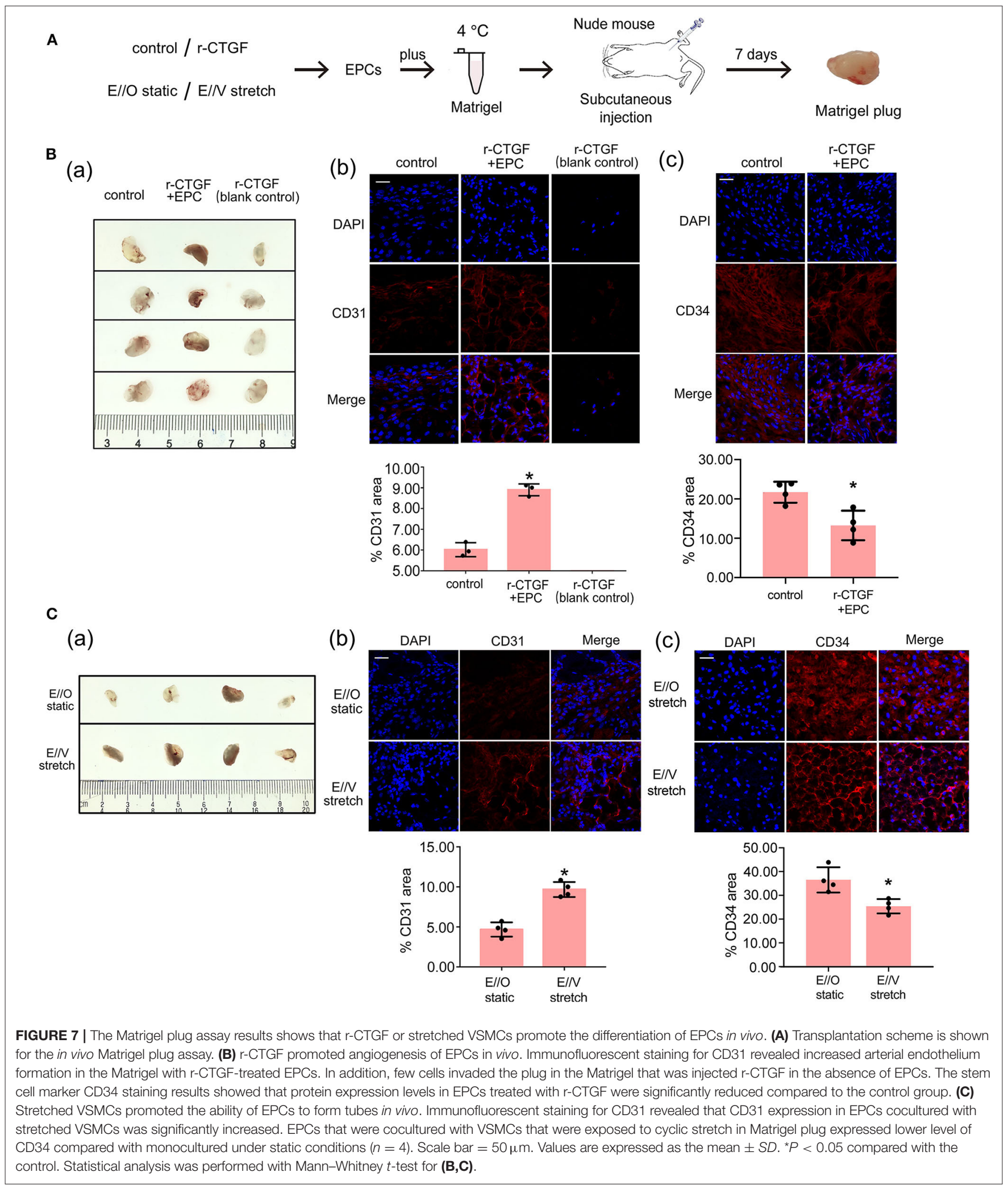

FZD8 was regulated by CTGF via transcriptional regulation (Supplementary Figure 3). We also further examined the effect of FZD8 loss of function or Wnt signaling in EPCs to confirm whether they are important for EPC differentiation. Our results indicated that FZD8/ $\beta$-catenin is vital for EPC differentiation and angiogenic activities. 


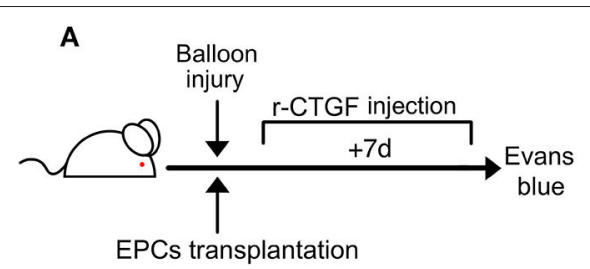

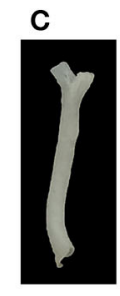

D

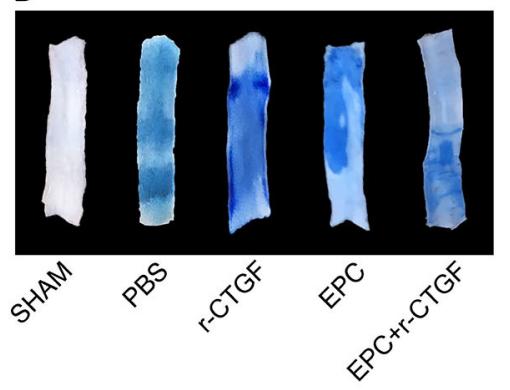

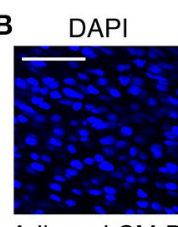
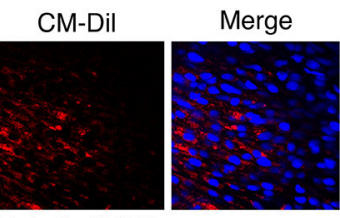

Adhered CM-Dil labelled EPCs

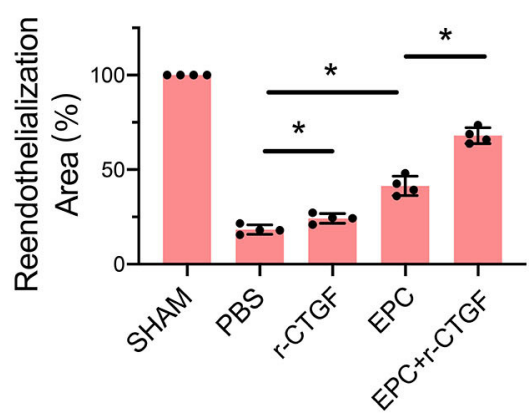

FIGURE 8 | EPC and r-CTGF promoted vascular intimal injury repair. (A) Schematic diagrams of the animal experimental protocol. (B) CM-Dil-labeled EPCs (red) adhered to the vascular lesion in situ. (C) The image of rat left carotid artery. (D) Intimal injury site was incubated with or without EPCs for 25-30 min, and injection of $\mathrm{r}$-CTGF $(2 \mu \mathrm{g} / \mathrm{kg} /$ day) promoted vascular repair. Reendothelialization area was observed by Evans blue staining after 7 days of the surgery. The percentage of the white area indicated the degree of reendothelialization. Scale bar $=50 \mu \mathrm{m}$. Values are expressed as the mean $\pm S D$. ${ }^{*} P<0.05$ compared with the control. Statistical analysis was performed with Mann-Whitney t-test for D.

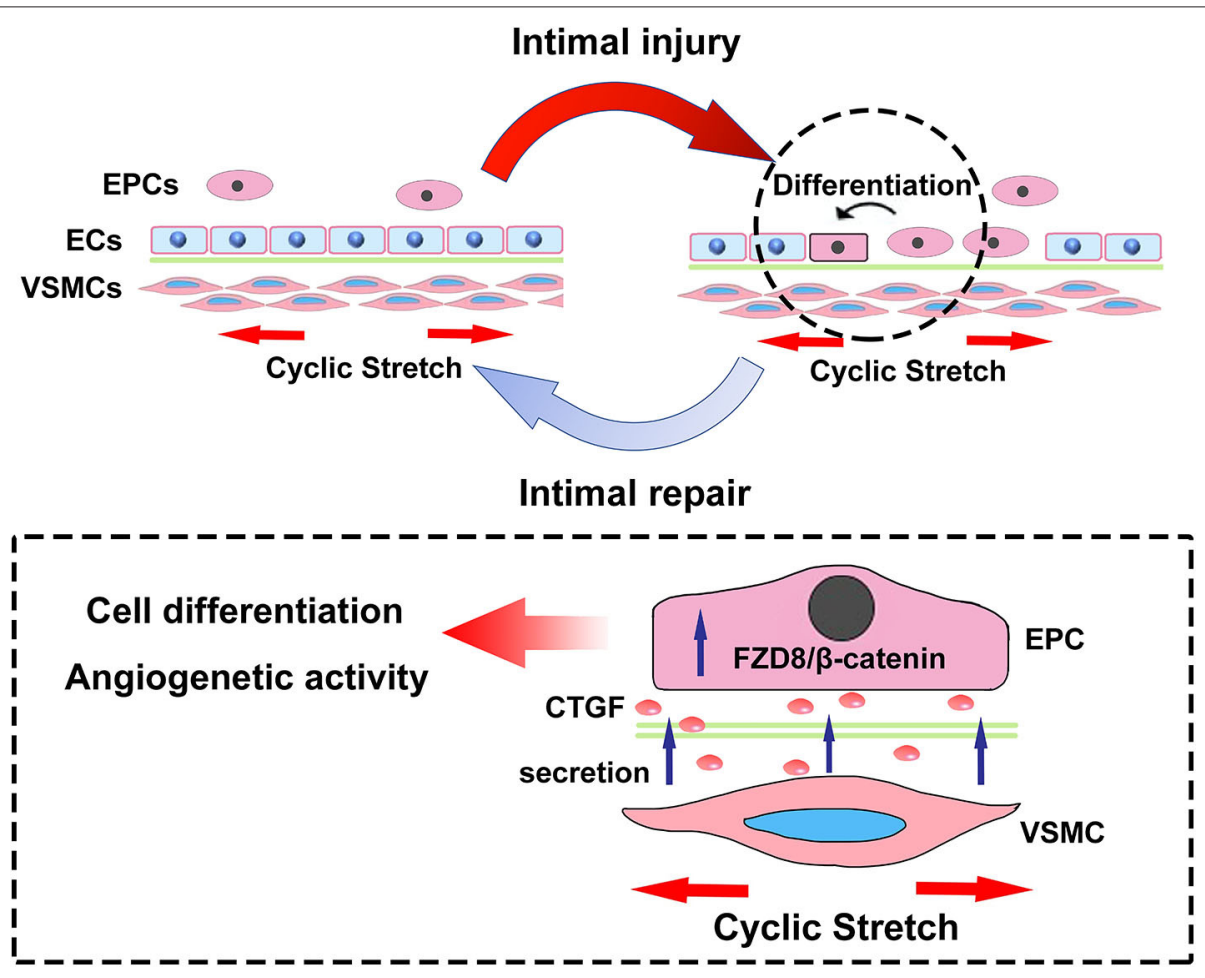

FIGURE 9 | The scheme here depicts the microenvironmental roles of VSMCs under cyclic stretch in EPC differentiation and angiogenesis during vascular repair. Cyclic stretch promotes the secretion of CTGF from VSMCs, which subsequently activates the FZD8/ $\beta$-catenin signaling pathway in EPCs, and increased $\beta$-catenin nuclear translocation eventually induces the differentiation and angiogenic abilities of EPCs. 
In conclusion, the present results revealed critical microenvironmental roles for VSMCs in the differentiation and angiogenesis process of EPCs during vascular injury. As is shown in Figure 9, physiological cyclic stretch (5\%) increased the secretion of CTGF from VSMCs. Secreted CTGF activated FZD8 in EPCs, which subsequently promoted $\beta$-catenin nuclear translocation from the cytoplasm and finally encouraged EPC differentiation fate toward ECs with enhanced angiogenic ability and reendothelialization capacity. In vivo study also indicated that stretched VSMCs induced cocultured EPC differentiation toward ECs; furthermore, r-CTGF enhanced angiogenesis and improved EPC reendothelialization capacity. The CTGF/FZD8/ $\beta$-catenin signaling axis may become a promising therapeutic target for vascular repair.

\section{DATA AVAILABILITY STATEMENT}

The original contributions presented in the study are included in the article/Supplementary Materials, further inquiries can be directed to the corresponding author.

\section{ETHICS STATEMENT}

The animal study was reviewed and approved by the Animal Research Committee of Shanghai Jiao Tong University.

\section{AUTHOR CONTRIBUTIONS}

JY: planned, performed, and analyzed animal studies and some of the cell and microscopy studies, prepared figures, and wrote part of the manuscript. W-BW: planned, performed, and analyzed the cell and microscopy studies, prepared figures, and wrote part of the manuscript. Y-JF: contributed to the immunofluorescence

\section{REFERENCES}

Armulik, A., Abramsson, A., and Betsholtz, C. (2005). Endothelial/pericyte interactions. Circ. Res. 97, 512-523. doi: 10.1161/01.RES.0000182903.16652.d7

Asahara, T., Murohara, T., Sullivan, A., Silver, M., Zee, R. V. D., Li, T., et al. (1997). Isolation of putative progenitor endothelial cells for angiogenesis. Science 275, 964-966. doi: 10.1126/science.275.5302.964

Asanuma, K., Magid, R., Johnson, C., Nerem, R. M., and Galis, Z. S. (2003). Uniaxial strain upregulates matrix-degrading enzymes produced by human vascular smooth muscle cells. Am. J. Physiol. Heart Circ. Physiol. 284, H1778H1784. doi: 10.1152/ajpheart.00494.2002

Blomme, B., Deroanne, C., Hulin, A., Lambert, C., Defraigne, J. O., Nusgens, B., et al. (2019). Mechanical strain induces a pro-fibrotic phenotype in human mitral valvular interstitial cells through RhoC/ROCK/MRTFA and Erk1/2 signaling pathways. J. Mol. Cell Cardiol. 135, 149-159. doi: 10.1016/j.yjmcc.2019.08.008

Chakravarthi, B., Chandrashekar, D. S., and Hodigere, B. S. A. (2018). Wnt receptor Frizzled 8 is a target of ERG in prostate cancer. Prostate 78, 1311-1320. doi: 10.1002/pros.23704

Chen, L. J., Wei, S. Y., and Chiu, J. J. (2013). Mechanical regulation of epigenetics in vascular biology and pathobiology. J. Cell. Mol. Med. 17, 437-448. doi: $10.1111 /$ jcmm.12031

Cheng, B. B., Qu, M. J., Wu, L. L., Shen, Y., Yan, Z. Q., Zhang, P., et al. (2014). MicroRNA-34a targets Forkhead box j2 to modulate differentiation of staining studies and the supplementary experiments in this project. HB: contributed to some of the animal studies. NL: contributed to the initial studies in this project. Q-PY, Y-LH, Z-LJ, Y-XQ, and YH: contributed to experimental facilities and reagents in this project. $\mathrm{YH}$ : planned and supervised the study and contributed to the writing of the final manuscript. The first draft of the manuscript was written by W-BW, JY, and YH. All authors contributed to the study conception and design, read, and approved the final manuscript.

\section{FUNDING}

This study was supported by grants from the National Natural Science Foundation of China (Nos. 11572198 and 11625209).

\section{ACKNOWLEDGMENTS}

A part of the abstract previously appeared as a conference abstract in the International Society for Heart Research (ISHR) world congress 2019.

\section{SUPPLEMENTARY MATERIAL}

The Supplementary Material for this article can be found online at: https://www.frontiersin.org/articles/10.3389/fcell.2020. 606989/full\#supplementary-material

Supplementary Figure 1 | VSMC identities are confirmed. Staining with $\alpha-S M A$ (green) revealed that the positively stained cells were VSMCs. Scale bar $=100 \mu \mathrm{m}$

Supplementary Figure 2 | Transfection with an FZD8 siRNA significantly decreased the FZD8 mRNA level $(n=3)$. Values are expressed as the mean \pm $S D .{ }^{*} P<0.05$ compared with the control.

Supplementary Figure $\mathbf{3}$ | The mRNA expression level of FZD8 in EPCs was stimulated by $r$-CTGF $(n=4)$. Values are expressed as the mean $\pm S D$. ${ }^{*} P<0.05$ compared with the control. endothelial progenitor cells in response to shear stress. J. Mol. Cell. Cardiol. 74, 4-12. doi: 10.1016/j.yjmcc.2014.04.016

Chien, S. (2007). Mechanotransduction and endothelial cell homeostasis: the wisdom of the cell. Am. J. Physiol. Heart Circ. Physiol. 292, H1209-H1224. doi: 10.1152/ajpheart.01047.2006

Cicha, I., Yilmaz, A., Klein, M., Raithel, D., Brigstock, D. R., Daniel, W. G., et al. (2005). Connective tissue growth factor is overexpressed in complicated atherosclerotic plaques and induces mononuclear cell chemotaxis in vitro. Arterioscler Thromb. Vasc. Biol. 25, 1008-1013. doi: 10.1161/01.ATV.0000162173.27682.7b

Dai, L., Li, C., Shedden, K. A., Misek, D. E., and Lubman, D. M. (2009). Comparative proteomic study of two closely related ovarian endometrioid adenocarcinoma cell lines using cIEF fractionation and pathway analysis. Electrophoresis 30, 1119-1131. doi: 10.1002/elps.200800505

Deanfield, J. E., Halcox, J. P., and Rabelink, T. J. (2007). Endothelial function and dysfunction: testing and clinicalrelevance. Circulation 115, 1285-1295. doi: 10.1161/CIRCULATIONAHA.106.652859

Deng, L., Blanco, F. J., Stevens, H., Lu, R., Caudrillier, A., McBride, M., et al. (2015). MicroRNA-143 activation regulates smooth muscle and endothelial cell crosstalk in pulmonary arterial hypertension. Circ. Res. 117, 870-883. doi: 10.1161/CIRCRESAHA.115.306806

Foubert, P., Squiban, C., Holler, V., Buard, V., Dean, C., Levy, B., et al. (2015). Strategies to enhance the efficiency of endothelial progenitor cell therapy by ephrin B2 pretreatment and coadministration with smooth muscle 
progenitor cells on vascular function during the wound-healing process in irradiated or nonirradiated condition. Cell Transplant. 24, 1343-1361. doi: 10.3727/096368913X672064

Ge, Q., Zhang, H., Hou, J., Wan, L., Cheng, W., Wang, X., et al. (2018). VEGF secreted by mesenchymal stem cells mediates the differentiation of endothelial progenitor cells into endothelial cells via paracrine mechanisms. Mol. Med. Rep. 17, 1667-1675. doi: 10.3892/mmr.2017.8059

Griese, D. P., Achatz, S., Batzlsperger, C. A., Strauch, U. G., Grumbeck, B., Weil, J., et al. (2003). Vascular gene delivery of anticoagulants by transplantation of retrovirally-transduced endothelial progenitor cells. Cardiovasc. Res. 58, 469-477. doi: 10.1016/S0008-6363(03)00266-9

Han, J. K., Kim, B. K., Won, J. Y., Shin, Y., Choi, S. B., Hwang, I., et al. (2016). Interaction between platelets and endothelial progenitor cells via LPA-Edg2 axis is augmented by PPAR- $\delta$ activation. J. Mol. Cell Cardiol. 97, 266-277. doi: 10.1016/j.yjmcc.2016.06.002

Hristov, M., Erl, W., and Weber, P. C. (2003). Endothelial progenitor cells: mobilization, differentiation, and homing. Arterioscler Thromb. Vasc. Biol. 23, 1185-1189. doi: 10.1161/01.ATV.0000073832.49290.B5

Hur, J., Yoon, C. H., Kim, H. S., Choi, J. H., Kang, H. J., Hwang, K. K., et al. (2004). Characterization of two types of endothelial progenitor cells and their different contributions to neovasculogenesis. Arterioscler Thromb. Vasc. Biol. 24, 288-293. doi: 10.1161/01.ATV.0000114236.77009.06

Joo, H. J., Seo, H. R., Jeong, H. E., Choi, S. C., Park, J. H., Yu, C. W., et al. (2014). Smooth muscle progenitor cells from peripheral blood promote the neovascularization of endothelial colony-forming cells. Biochem. Bioph. Res. Co. 449, 405-411. doi: 10.1016/j.bbrc.2014.05.061

Jun, J. I., and Lau, L. F. (2011). Taking aim at the extracellular matrix: CCN proteins as emerging therapeutic. targets. Nat. Rev. Drug Discov. 10, 945-963. doi: $10.1038 / \mathrm{nrd} 3599$

Kelly, M., Joseph, C. G., Andrew, P. V., Megan, J. R., Dalyz, O., Ian, C. H., et al. (2020). Stepwise differentiation and functional characterization of human induced pluripotent stem cell-derived choroidal endothelial cells. Stem Cell. Res. Ther. 11, 409. doi: 10.1186/s13287-020-01903-4

Kubota, S., and Takigawa, M. (2007). CCN family proteins and angiogenesis: from embryo to adulthood. Angiogenesis 10, 1-11. doi: 10.1007/s10456-006-9058-5

Kuliszewski, M. A., Fujii, H., Liao, C., Smith, A. H., Xie, A., Lindner, J. R., et al. (2009). Molecular imaging of endothelial progenitor cell engraftment using contrast-enhanced ultrasound and targeted microbubbles. Cardiovasc. Res. 83, 653-662. doi: 10.1093/cvr/cvp218

Kundi, R., Hollenbeck, S. T., Yamanouchi, D., Herman, B. C., Edlin, R., Ryer, E. J., et al. (2009). Arterial gene transfer of the TGF-beta signalling protein Smad3 induces adaptive remodelling following angioplasty: a role for CTGF. Cardiovasc. Res. 84, 326-335. doi: 10.1093/cvr/cvp220

Li, H., Zhang, X. Y., Guan, X. M., Cui, X. D., Wang, Y. L., Chu, H. R., et al. (2012). Advanced glycation end products impair the migration, adhesion and secretion potentials of late endothelial progenitor cells. Cardiovasc. Diabetol. 11:46. doi: 10.1186/1475-2840-11-46

Li, L., Liu, H., Xu, C., Deng, M., Song, M., Yu, X., et al. (2017). VEGF promotes. endothelial progenitor cell differentiation and vascular repair through connexin 43. Stem Cell Res. Ther. 8:237. doi: 10.1186/s13287-017-0684-1

Li, N., Wang, W. B., Bao, H., Shi, Q., Jiang, Z. L., Qi, Y. X., et al. (2018). MicroRNA-129-1-3p. regulates cyclic stretch-induced endothelial progenitor cell differentiation by targeting Runx2. J. Cell. Biochem. 120, 5256-5267. doi: $10.1002 / j$ cb. 27800

Li, X., Pongkitwitoon, S., Lu, H., Lee, C., Gelberman, R., and Thomopoulos, S. (2019). CTGF induces tenogenic differentiation and proliferation of adipose-derived stromal cells. J. Orthop. Res. 37, 574-582. doi: 10.1002/jor. 24248

Liu, S. C., Chuang, S. M., Hsu, C. J., Tsai, C. H., Wang, S. W., and Tang, C. H. (2014). CTGF increases. vascular endothelial growth factor-dependent angiogenesis in human synovial fibroblasts by increasing miR-210 expression. Cell Death Dis. 5:e1485. doi: 10.1038/cddis.2014.453

Liu, X., McBride, J., Zhou, Y., Liu, Z., and Ma, J. X. (2013). Regulation of endothelial progenitor cell. release by Wnt signaling in bone marrow. Invest. Ophthalmol. Vis. Sci. 54, 7386-7394. doi: 10.1167/iovs.13-13163

Majidinia, M., Aghazadeh, J., Jahanban-Esfahlani, R., and Yousefi, B. (2018). The roles of $\mathrm{Wnt} /$ beta-catenin pathway in tissue development and regenerative medicine. J. Cell. Physiol. 233, 5598-5612. doi: 10.1002/jcp. 26265
Malinda, K. M. (2009). In vivo matrigel migration and angiogenesis assay. Methods Mol. Biol. 467, 287-294. doi: 10.1007/978-1-59745-241-0_17

Maul, T. M., Chew, D. W., Nieponice, A., and Vorp, D. A. (2011) Mechanical stimuli differentially control stem cell behavior: morphology, proliferation, and differentiation. Biomech. Model Mechanobiol. 10, 939-953. doi: 10.1007/s10237-010-0285-8

Miyake, Y., Furumatsu, T., Kubota, S., Kawata, K., Ozak, T., and Takigawa, M. (2011). Mechanical stretch. increases CCN2/CTGF expression in anterior cruciate ligament-derived cells. Biochem. Biophys. Res. Commun. 409, 247-252. doi: 10.1016/j.bbrc.2011.04.138

Murasawa, S., Kawamoto, A., Horii, M., Nakamori, S., and Asahara, T. (2005). Niche-dependent translineage commitment of endothelial progenitor cells, not cell fusion in general, into myocardial lineage cells. Arterioscler Thromb. Vasc. Biol. 25, 1388-1394. doi: 10.1161/01.ATV.0000168409.69 960.e9

Murillo-Garzon, V., Gorrono-Etxebarria, I., and Akerfelt, M. (2018). Frizzled8 integrates Wnt-11 and transforming growth factor-beta signaling in prostate cancer. Nat. Commun. 9:1747. doi: 10.1038/s41467-01804042-w

Ostriker, A., Horita, H. N., Poczobutt, J., Weiser-Evans, M. C., and Nemenoff, R. A. (2014). Vascular smooth muscle cell-derived transforming growth factor-beta promotes maturation of activated, neointima lesion-like macrophages. Arterioscler Thromb. Vasc. Biol. 34, 877-886. doi: 10.1161/ATVBAHA.114.303214

Qi, Y. X., Jiang, J., Jiang, X. H., Wang, X. D., Ji, S. Y., Han, Y., et al. (2011). PDGF$\mathrm{BB}$ and TGF- $\beta 1$ on cross-talk between endothelial and smooth muscle cells in vascular remodeling induced by low shear stress. Proc. Natl. Acad. Sci. U.S.A. 108, 1908-1913. doi: 10.1073/pnas.1019219108

Qi, Y. X., Qu, M. J., Long, D. K., Liu, B., Yao, Q. P., Chien, S., et al. (2008). RhoGDP dissociation inhibitor alpha downregulated by low shear stress promotes vascular smooth muscle cell migration and apoptosis: a proteomic analysis. Cardiovasc. Res. 80, 114-122. doi: 10.1093/cvr/cvn158

Qi, Y. X., Qu, M. J., Yan, Z. Q., Zhao, D., Jiang, X. H., Shen, B. R., et al. (2010). Cyclic strain modulates migration and proliferation of vascular smooth muscle cells via Rho-GDIalpha, Rac1, and p38 pathway. J. Cell. Biochem. 109, 906-914. doi: $10.1002 / j \mathrm{cb} .22465$

Qi, Y. X., Yao, Q. P., Huang, K., Shi, Q., Zhang, P., Wang, G. L., et al. (2016). Nuclear envelope proteins modulate proliferation of vascular smooth muscle cells during cyclic stretch application. Proc. Natl. Acad. Sci. U.S.A. 113, 5293-5298. doi: 10.1073/pnas.1604569113

Shudo, Y., Cohen, J. E., Macarthur, J. W., Atluri, P., Hsiao, P. F., Yang, E. C., et al. (2013). Spatially oriented, temporally sequential smooth muscle cell-endothelial progenitor cell bi-level cell sheet neovascularizes ischemic myocardium. Circulation 128(11 Suppl. 1), S59-S68. doi: 10.1161/CIRCULATIONAHA.112.000293

Tripurani, S. K., Wang, Y., Fan, Y. X., Rahimi, M., Wong, L., Lee, M. H., et al. (2018). Suppression of $\mathrm{Wnt} /$ beta-catenin signaling by EGF receptor is required for hair follicle development. Mol. Biol. Cell. 29, 2784-2799. doi: 10.1091/mbc.E18-08-0488

Tu, J., Yang, F., Wan, J., Liu, Y., Zhang, J., Wu, B., et al. (2014). Lightcontrolled. astrocytes promote human mesenchymal stem cells toward neuronal differentiation and improve the neurological deficit in stroke rats. Glia 62, 106-121. doi: 10.1002/glia.22590

Wachowiak, M. P., Hay, D. C., and Johnson, M. J. (2016). Assessing heart rate variability through wavelet-based. statistical measures. Comput. Biol. Med. 77, 222-230. doi: 10.1016/j.compbiomed.2016.07.008

Wang, W. B., Li, H. P., Yan, J., Zhuang, F., Bao, M., Liu, J. T., et al. (2019). CTGF regulates cyclic stretch-induced vascular smooth muscle cell proliferation via microRNA-19b-3p. Exp. Cell. Res. 376, 77-85. doi: 10.1016/j.yexcr.2019.01.015

Wei, S., Huang, J., Li, Y., Zhao, J., Luo, Y., Meng, X., et al. (2015). Novel zinc finger transcription factor ZFP580 promotes differentiation of bone marrow-derived endothelial progenitor cells into endothelial cells via eNOS/NO pathway. J. Mol. Cell. Cardiol. 87, 17-26. doi: 10.1016/j.yjmcc.2015. 08.004

Yan, J., Bao, H., Fan, Y. J., Jiang, Z. L., Qi, Y. X., and Han, Y. (2020). Platelet-derived microvesicles promote endothelial progenitor cell proliferation in intimal injury by delivering TGF- $\beta 1$. FEBS J. doi: 10.1111/febs.15293. [Epub ahead of print] 
Yao, Q. P., Xie, Z. W., Wang, K. X., Zhang, P., Han, Y., Qi, Y. X., et al. (2017). Profiles of long. noncoding RNAs in hypertensive rats: long noncoding RNA XR007793 regulates cyclic strain-induced proliferation and migration of vascular smooth muscle cells. J. Hypertens. 35, 1195-1203. doi: 10.1097/HJH.0000000000001304

Yu, Y., Gao, Y., Qin, J., Kuang, C. Y., Song, M. B., Yu, S. Y., et al. (2010). CCN1 promotes the differentiation of endothelial progenitor cells and reendothelialization in the early phase after vascular injury. Basic Res. Cardiol. 105, 713-724. doi: 10.1007/s00395-010-0117-0

Zhang, M., Malik, A. B., and Rehman, J. (2014). Endothelial progenitor cells and vascular repair. Curr. Opin. Hematol. 21, 224-228. doi: 10.1097/MOH.0000000000000041
Conflict of Interest: The authors declare that the research was conducted in the absence of any commercial or financial relationships that could be construed as a potential conflict of interest.

Copyright (C) 2020 Yan, Wang, Fan, Bao, Li, Yao, Huo, Jiang, Qi and Han. This is an open-access article distributed under the terms of the Creative Commons Attribution License (CC BY). The use, distribution or reproduction in other forums is permitted, provided the original author(s) and the copyright owner(s) are credited and that the original publication in this journal is cited, in accordance with accepted academic practice. No use, distribution or reproduction is permitted which does not comply with these terms. 